\title{
Vasopressin Differentially Modulates Non-NMDA Receptors in Vasopressin and Oxytocin Neurons in the Supraoptic Nucleus
}

\author{
Michiru Hirasawa, ${ }^{1}$ Didier Mouginot, ${ }^{2}$ Michael G. Kozoriz, ${ }^{1}$ Samuel B. Kombian, ${ }^{3}$ and Quentin J. Pittman ${ }^{1}$ \\ ${ }^{1}$ Neuroscience Research Group, Department of Physiology and Biophysics, University of Calgary, Calgary, Alberta, T2N 4N1 Canada, ${ }^{2}$ Centre de Recherché \\ en Neurosciences, Centre Hospitalier de l'Université Laval, Sainte-Foy, Quebec, G1Y 4G2 Canada, and 'Faculty of Pharmacy, Kuwait University, Safat, 13110 \\ Kuwait
}

\begin{abstract}
Magnocellular neurons of the supraoptic nucleus release the neuropeptides oxytocin and vasopressin from their dendrites to regulate their synaptic inputs. This study aims to determine the cellular mechanism by which vasopressin modulates excitatory synaptic transmission. Presumably by electroporation through perforated patch, we were able to successfully introduce biocytin into cells in which we performed an electrophysiological study. This method enabled us to determine that roughly half of the recorded neurons were immunoreactive to oxytocin-associated neurophysin and showed two characteristic features: an inward rectification and a sustained outward rectification. The remaining half showed a linear voltage-current relationship and was immunoreactive to vasopressin-associated neurophysin. Using these electrophysiological characteristics and post hoc immunohistochemistry to identify vasopressin or oxytocin neurons, we found that vasopressin decreased evoked EPSCs in vasopressin neurons while increasing EPSCs in oxytocin neurons. In both types of neurons, EPSC decay constants were not affected, indicating that desensitization of non-NMDA receptors did not underlie the EPSC amplitude change. In vasopressin neurons, both vasopressin and a V1a receptor agonist, F-180, decreased AMPA-induced currents, an effect blocked by a V1a receptor antagonist SR49059. In oxytocin neurons, AMPA-induced currents were facilitated by vasopressin, whereas F-180 had no effect. An oxytocin receptor antagonist blocked the facilitatory effect of vasopressin. Thus, we conclude that vasopressin inhibits EPSCs in vasopressin neurons via postsynaptic V1a receptors, whereas it facilitates EPSCs in oxytocin neurons through oxytocin receptors.
\end{abstract}

Key words: EPSC; non-NMDA receptor; V1a receptor; oxytocin receptor; vasopressin; supraoptic nucleus; dendritic release

\section{Introduction}

It is now well accepted that dendrites are not merely recipients of synaptic information but also can be the active speaker at the synapse by releasing neurotransmitters. Magnocellular neurons (MCNs) of the supraoptic nucleus (SON) are one of the cell groups shown to be capable of dendritic release of neurotransmitters. MCNs release arginine-vasopressin (AVP) and oxytocin (OXT) within the SON from their dendrites and somata (Moos et al., 1984; Mason et al., 1986; Pow and Morris, 1989) to control their own electrical and secretory activity (for review, see Leng et al., 1999). AVP inhibits the phasic pattern of action potential firing characteristic of AVP neurons (Ludwig and Leng, 1998). Furthermore, this inhibitory action seems to be correlated with the level of electrical activity of the individual AVP neuron (Gouzenes et al., 1998). In particular, AVP has been found to tune the phasic pattern of action potential firing characteristic of AVP neurons (Gouzenes et al., 1998; Ludwig and Leng, 1998). Previous studies in our laboratory showed that neuropeptides synthe-

\footnotetext{
Received 0ct. 31, 2002; revised Feb. 12, 2003; accepted Feb. 26, 2003.

This study was supported by the Canadian Institute for Health Research (CIHR), by personal awards from the Heart and Stroke Foundation of Canada/CIHR (M.H.) and the CIHR/Canadian Hypertension Society (D.M.), by Kuwait University Grant FDT 113 (S.B.K.), and by the Alberta Heritage Foundation for Medical Research (Q.J.P.). We thank Lorenzo Bauce and Mio Tsutsui for the technical assistance.

Correspondence should be addressed to Michiru Hirasawa, Department of Physiology and Biophysics, University of Calgary, 3330 Hospital Drive Northwest, Calgary, Alberta, T2N 4N1 Canada. E-mail: hirasawa@ucalgary.ca. Copyright $\odot 2003$ Society for Neuroscience $\quad$ 0270-6474/03/234270-08\$15.00/0
}

sized and released from MCNs, namely OXT and AVP, modulate excitatory synaptic input onto themselves. OXT suppresses EPSCs by acting at the presynaptic terminals (Kombian et al., 1997). This effect is through the inhibition of presynaptic calcium channels, resulting in reduction of glutamate release (Hirasawa et al., 2001). In contrast, OXT inhibits $\mathrm{GABA}_{\mathrm{A}}$ receptor-mediated IPSCs through an interaction of OXT and $\mathrm{GABA}_{\mathrm{A}}$ receptors in the postsynaptic cell (Brussaard et al., 1996). AVP has been shown previously to inhibit evoked EPSCs in this nucleus (Kombian et al., 2000), but the cellular mechanism of this effect awaits determination.

Glutamatergic transmission in the SON is modulated by numerous neurotransmitters, including GABA, adenosine, histamine, and glutamate itself via metabotropic glutamate receptors (Kombian et al., 1997; Oliet and Poulain, 1999; Li and Hatton, 2000; Schrader and Tasker, 1997). The afferent neurons containing these neurotransmitters transfer peripheral or central information from various brain areas by modulating the tone of excitatory input to the SON. In contrast, AVP and OXT comprise a micro-feedback loop within the nucleus for controlling synaptic inputs, thus representing a mechanism of direct monitoring and control of MCN activity at a different level. Therefore, to better understand how MCNs determine their activity level and the amount of neuropeptide output, it is important to resolve how these neuropeptides modulate synaptic inputs onto themselves. 
The goal of this study is to investigate the cellular mechanism of AVP action to modulate excitatory synaptic inputs into the SON.

\section{Materials and Methods}

All experiments were performed in accordance with the guidelines established by the Canadian Council on Animal Care and were approved by the University of Calgary Animal Care Committee. Attention was paid to use only the number of animals necessary to produce reliable results.

Slice preparation. Adult male Sprague Dawley rats (150-250 gm) were decapitated under halothane anesthesia, the brain was removed, and coronal slices $300-400 \mu \mathrm{m}$ thick containing the SON were generated at $0-2^{\circ} \mathrm{C}$ in a low-Ca ${ }^{2+}$, low- $\mathrm{Na}^{+}$-containing buffer solution of composition (in mM): $87 \mathrm{NaCl}, 2.5 \mathrm{KCl}, 1.25 \mathrm{NaH}_{2} \mathrm{PO}_{4}, 7 \mathrm{MgCl}_{2}, 0.5 \mathrm{CaCl}_{2}, 25$ $\mathrm{NaHCO}_{3}, 25$ glucose, and 20 sucrose. Slices were then incubated at room temperature $\left(22^{\circ} \mathrm{C}\right)$ for at least $1 \mathrm{hr}$ in artificial CSF (ACSF) of the following composition (in mM): $126 \mathrm{NaCl}, 2.5 \mathrm{KCl}, 1.2 \mathrm{NaH}_{2} \mathrm{PO}_{4}, 1.2$ $\mathrm{MgCl}_{2}, 2.4 \mathrm{CaCl}_{2}, 18 \mathrm{NaHCO}_{3}$, and 11 glucose. Both solutions were bubbled continuously with a mixture of $95 \% \mathrm{O}_{2}, 5 \% \mathrm{CO}_{2}$.

Electrophysiological recording. A hemisected slice was transferred into a recording chamber, where it was submerged and perfused at $30-32^{\circ} \mathrm{C}$ with ACSF. The nystatin-perforated patch technique was used for the recording with electrodes having a tip resistance of 3-7 M $\Omega$. Whole-cell access was attained within 2-30 min with a series/access resistance of 10-40 $\mathrm{M} \Omega$. The internal recording solution contained (in $\mathrm{mM}$ ): 120 $\mathrm{K}$-acetate, $5 \mathrm{MgCl}_{2}, 10 \mathrm{EGTA}$, and 40 HEPES. Nystatin was dissolved in DMSO with Pluronic F127 and added to the internal solution to yield a final concentration of $450 \mu \mathrm{g} / \mathrm{ml}$. The $\mathrm{pH}$ was adjusted to 7.3. All experiments were done on MCNs voltage-clamped at $-80 \mathrm{mV}$ using an Axopatch 1D or Axopatch 200A amplifier and pClamp 7 software (Axon Instruments, Foster City, CA). MCNs were identified on the basis of the delayed onset to action potential generation in response to positive current injection (Tasker et al., 1991; Armstrong, 1995). Membrane currents were recorded without series resistance compensation, filtered at $1 \mathrm{kHz}$, digitized at $5-10 \mathrm{kHz}$, and stored for off-line analysis. A $20 \mathrm{mV}$ hyperpolarizing pulse lasting for $75 \mathrm{msec}$ was applied regularly throughout each experiment, and the steady-state current and decay rate $(\tau)$ of the capacitance transient were monitored as measures of input resistance and series/access resistance, respectively. Cells that showed $>15 \%$ change in these parameters were excluded from additional analysis. Hard copy chart records were also captured on a Gould recorder (Gould Instruments, Valley View, OH).

Synaptic currents were evoked in the MCNs with a bipolar stimulating electrode placed in the hypothalamic region dorsomedial to the SON, close to the optic tract. For all experiments, picrotoxin $50 \mu \mathrm{M}$ was added to ACSF to block $\mathrm{GABA}_{\mathrm{A}}$ receptor-mediated chloride currents yielding pharmacologically isolated EPSCs. As reported previously, EPSCs were mediated by non-NMDA receptors, because they were sensitive to $10 \mu \mathrm{M}$ DNQX, a non-NMDA receptor antagonist (Kombian et al., 1996). Stimulus intensities that yielded $50-60 \%$ of the maximum EPSC amplitude were used in the present study.

The amplitude of EPSCs was taken as a measure of the magnitude of synaptic strength, and the amplitude of the current induced by a brief bath application of AMPA was taken as a measure of the postsynaptic efficacy of the drug. Repeated application of AMPA did not attenuate the response, and two or more AMPA currents were averaged for all conditions. The current amplitudes were expressed as mean \pm SEM of the percentage change from control values. Only cells that showed significant recovery $(>70 \%)$ from any drug effect were included in the data analysis. Changes $>15 \%$ of control value were considered to be effective. Statistical comparisons were performed by using appropriate tests, i.e., unpaired and paired Student's $t$ tests, and the least-squares method was used for linear regression. A value of $p<0.05$ was considered significant.

Immunohistochemistry. For immunohistochemical identification of the recorded cell, biocytin $(1-1.5 \mathrm{mg} / \mathrm{ml})$ was included in the internal solution, which was filled into the cell at the end of the experiment by applying adequate positive currents (in current-clamp mode) to make the cell fire action potentials at $30-50 \mathrm{~Hz}$ for $4-5 \mathrm{~min}$. On termination of the recording, the slice was fixed in $4 \%$ paraformaldehyde in $0.1 \mathrm{M} \mathrm{PBS}$ at a pH of 7.4 for $24 \mathrm{hr}$, transferred to $20 \%$ sucrose-PBS, then cut into 15 $\mu \mathrm{m}$ sections. Alternate sections were treated with either OXTneurophysin mouse monoclonal antibody (CRL-1800; American Type Culture Collection, Manassas, VA) (Ben Barak et al., 1985) or AVPneurophysin mouse monoclonal antibody (CRL-1799; American Type Culture Collection) (Ben Barak et al., 1985), both at 1:2000 dilution in 0.1 м PBS with $10 \%$ BSA, $0.1 \%$ Triton-X, and incubated overnight at room temperature. After the slices had been washed with PBS, a 1:500 dilution of cyanine 3-conjugated goat anti-mouse secondary antibody (Amersham Pharmacia Biotech, Little Chalfont, UK) was added with a 1:500 dilution of avidin-conjugated fluorescein isothiocyanate (FITC; Vector Laboratories, Burlingame, CA) to label biocytin for $2 \mathrm{hr}$ at room temperature. The sections were examined under a confocal fluorescence microscope for the presence of OXT- or AVP-neurophysin immunoreactivity (cyanine 3) and biocytin labeling (FITC). When a biocytin-labeled cell was not observed in more than two serial sections, phenotypes of the labeled cells were identified on the basis of a positive reaction for one peptide or a negative reaction when nearby neurons were clearly stained.

Chemical compounds. All substances were prepared as $1000 \times$ stock solutions and diluted to their final concentration in ACSF just before use. AVP and [des-glycinamide ${ }^{9}, d\left(\mathrm{CH}_{2}\right)_{5}{ }^{1}, \mathrm{O}-\mathrm{Me}-\mathrm{Tyr}^{2}, \mathrm{Tyr}^{4}, \mathrm{Orn}^{8}$ ]vasotocin (OXT antagonist) were purchased from Bachem (Torrence, CA). Picrotoxin, nystatin, and DMSO were from Sigma (St. Louis, MO). AMPA was obtained from Research Biochemicals (Natick, MA). F-180 was a kind gift from Ferring Research Institute (San Diego, CA), and SR49059 was kindly provided by Sanofi-Synthelabo Recherche (Toulouse, France).

\section{Results}

Previous work by Stern and Armstrong (1995) showed that a majority of MCNs immunopositive for OXT-associated neurophysin displayed a sustained outward rectification (SOR) that was largely absent in cells identified as AVP-associated neurophysin-immunoreactive MCNs. Because the SOR is activated at a depolarized membrane potential $(-40 \mathrm{mV})$ and inactivated with hyperpolarizing current steps, we were able to replicate their finding in our experimental protocol by initially clamping the cells at $-40 \mathrm{mV}$ and applying a series of voltage steps ranging from -130 to $-40 \mathrm{mV}$. As illustrated in Fig. 1, A1 and $A 2,53.2 \%(41 / 77)$ of the recorded MCNs did not show an SOR, as apparent from the linear current-voltage relationship. In contrast, in the remaining $46.8 \%(36 / 77)$ of the cells tested, an SOR was apparent at membrane potentials higher than $-70 \mathrm{mV}$ (Fig. 1 B1,B2). Interestingly, 80.6\% (29/36) of the cells presenting with an SOR also displayed a hyperpolarization-induced inward rectification (IR) at membrane potentials more negative than $-90 \mathrm{mV}$ (Fig. 1B1,B2), a current that was blocked by $2 \mathrm{~mm}$ external cesium (data not shown). Furthermore, the absence of SOR was also correlated with the absence of IR in 95.1\% (39/41) of these neurons. Those cells that showed only either SOR or IR were excluded from additional study. There was no significant difference in the input and access resistance between cells with or without IR. To further confirm the identity of these two groups of cells, post hoc immunohistological analysis was performed on a subset of the recorded cells. To identify the recorded neurons histologically, we have modified the single-cell labeling method used for dye filling a neuron simultaneously with juxtacellular recording (Pinault, 1996; Kirouac and Pittman, 1999). Biocytin was introduced successfully into the recorded neurons by tracer electroporation as described in Materials and Methods. All 12 cells that showed neither SOR nor IR were identified to be AVP neuron by post hoc immunohistochemistry (Fig. 1 A3). In contrast to this, the majority of the cells that showed both SOR and IR (five of seven) were immunohistochemically classified to be OXT neurons (Fig. 1B3). The remaining two cells were classified as AVP neurons. The strong correlation of the presence or absence 


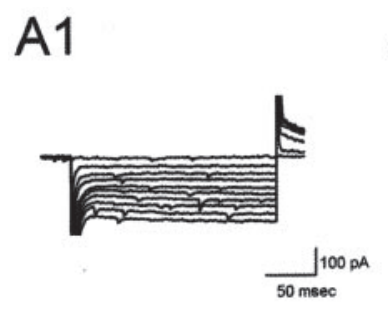

A2
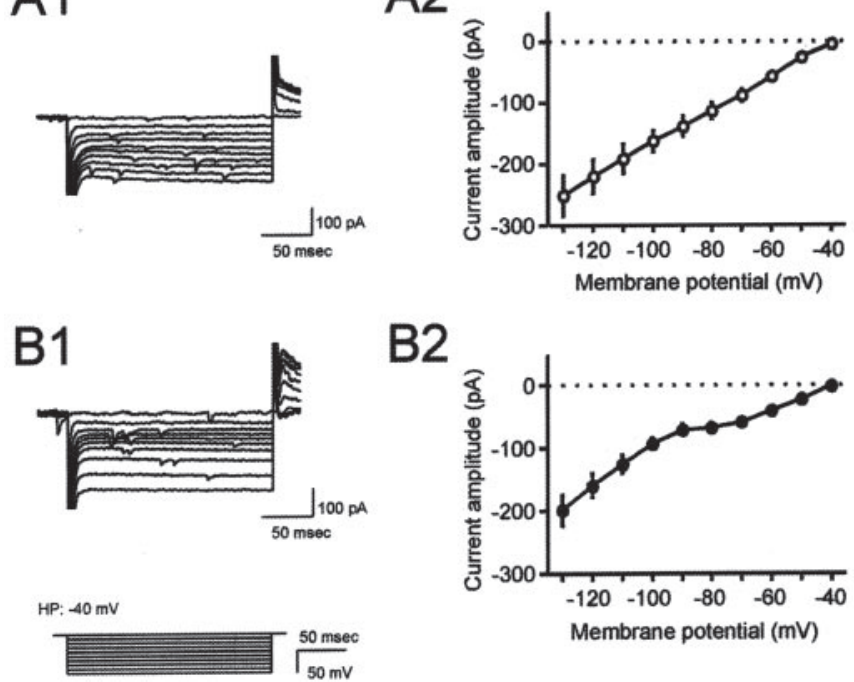

B2

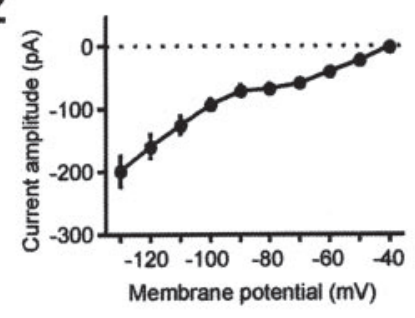

A3
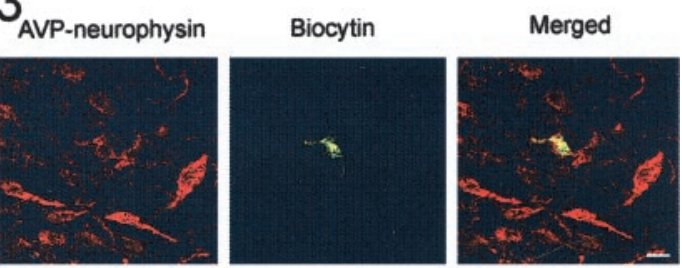

B3 OXT-neurophysin

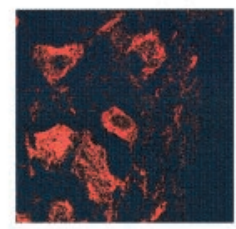

Biocytin
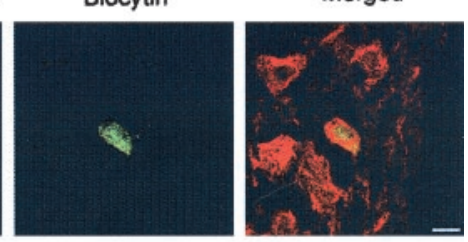

Figure 1. The presence or the absence of voltage-activated membrane currents characterize two distinct subpopulations of magnocellular neurons. A1, Superimposed current traces representative of a subpopulation of magnocellular neurons showing a linear $I-V$ relationship in response to $200 \mathrm{msec}$ hyperpolarizing voltage steps elicited from a holding potential of $-40 \mathrm{mV}$. The voltage paradigm is depicted at bottom left. $A 2$, A mean $I-V$ plot of this population $(n=10)$. $B 1$, A representative of another subpopulation of magnocellular neurons shows a different $I-V$ relationship in response to the same protocol. B2, The mean $I-V$ plot from 10 magnocellular neurons showing a similar nonlinear relationship. A sustained outward rectification (SOR) is observed at membrane potentials more positive than $-70 \mathrm{mV}$, whereas a hyperpolarization-induced inward rectification (IR) appears at membrane potential more negative than $-90 \mathrm{mV}$. $A 3$, Confocal image of a cell that did not display SOR/IR, filled with biocytin by electroporation through perforated patch (green). This cell was colabeled with AVP-associated neurophysin antibody (red). B3, Another cell that showed SOR/IR, which was filled with biocytin (green) and was OXT-associated neurophysin immunoreactive (red). Scale bars, $10 \mu \mathrm{m}$.

of SOR/IR to specific phenotypes of MCNs suggests that the phenotype of MCNs can be determined using these electrophysiological fingerprints. In the rest of the study, cells were classified as OXT or AVP neurons using these biophysical characteristics.

Our previous study had shown that AVP dose-dependently causes inhibition of EPSCs, with a maximum inhibition at a dose of $2 \mu \mathrm{M}$ (Kombian et al., 2000). In the present study, a maximum dose was used to induce a robust response and identify the cellular mechanism of its action. A bath application of $2 \mu \mathrm{M}$ AVP reversibly reduced the EPSC amplitude by $25.2 \pm 2.7 \%$ in 24 of 28 AVP neurons $(p<0.05)$ (Fig. $2 A 1, A 3)$. Of the remaining cells, one responded with an increase of $17.4 \%$, whereas the other three cells showed no change. This result is in agreement with our previous study (Kombian et al., 2000). A similar inhibitory effect on EPSCs was seen with bath application of a V1a receptorspecific agonist, F-180. At $2 \mu \mathrm{M}, \mathrm{F}-180$ reduced the EPSC amplitude in six of nine cells by $35.8 \pm 2.9 \%(p<0.05)$ (Fig. 2A2,A3).

In contrast to the AVP-induced inhibition of the EPSCs in AVP neurons, AVP $(2 \mu \mathrm{M})$ increased the EPSCs by $19.6 \pm 3.2 \%$ in six of eight OXT neurons $(p<0.05)$ (Fig. 2 B1,B3). The two remaining neurons showed either no response or a decrease (by $34 \%)$ in the EPSC amplitude. In this type of neuron, F-180 (2 $\mu \mathrm{M})$ did not change the size of EPSCs in the majority of the cells tested ( ix of eight cells) $(0.3 \pm 4.1 \%$ change; $p>0.05, n=6)$ (Fig. $2 \mathrm{~B} 2, \mathrm{B3})$, whereas the remaining two cells responded with an increase of $23.1 \%$ and $156.1 \%$. These results indicate that in AVP neurons, AVP decreases EPSCs via V1a receptors, whereas in OXT neurons, it increases EPSCs via another mechanism. There was no correlation between the initial EPSC amplitude and the direction of the response to AVP or F-180 $\left(R^{2}=0.03\right.$; slope deviation, $p>0.05)$. In both types of neurons, there was no significant change in input resistance or holding current.

Because the directions of the responses were correlated with the phenotype of the neurons, it is probable that the differences were a feature of the postsynaptic cell. Changes in EPSC ampli- tude can be a result of changes in non-NMDA receptor kinetics, such as desensitization rate or channel conductance. Changes in the desensitization rate of receptors would be reflected in the decay constant of EPSCs (Vyklicky et al., 1991). The decay constants between the two cell groups were similar (AVP neurons: $12.1 \pm 1.6 \mathrm{msec}, n=11$; OXT neurons: $11.5 \pm 0.8 \mathrm{msec}, n=10$; $p>0.05)$. In addition, the decay rate of the evoked EPSCs in both these cell phenotypes was not altered by AVP (AVP neurons: $-4.2 \pm 9.6 \%$ change, $n=5$; OXT neurons: $8.1 \pm 8.1 \%$ change, $n=4 ; p>0.05$ ) (Fig. 3). Thus, alteration in non-NMDA channel kinetics cannot account for the effects of AVP in these cells. Furthermore, F-180 also had no effect on the decay constant of EPSCs in either phenotype (AVP neurons: $-0.3 \pm 6.6 \%$ change, $n=6$; OXT neurons: $5.1 \pm 8.3 \%$ change, $n=6$; $p>0.05$ ).

To determine whether AVP caused an alteration in the steadystate sensitivity of the AMPA receptor-activated channels to alter the postsynaptic response to glutamate, AMPA was briefly bathapplied to activate non-NMDA receptors with a known concentration of the agonist. AMPA application $(5 \mu \mathrm{M}, 10 \mathrm{sec})$ induced a reproducible steady-state inward current. In AVP neurons, application of AVP reversibly reduced the amplitude of AMPA currents by $36.5 \pm 3.6 \%$ in 11 of 13 cells tested $(p<0.05)$ (Fig. $4 A, C)$. In the remaining two cells, AVP either had no effect or increased the AMPA current by 52.5\%, respectively. In OXT neurons, AVP induced reversible increases in the amplitude of AMPA currents by $33.1 \pm 14.2 \%$ in 11 of 14 cells tested $(p<$ 0.05) (Fig. 5A,C). AVP was without effect in the remaining three cells. These observed effects of AVP on AMPA-induced currents are in agreement with AVP effects on evoked EPSCs.

To determine whether both of these contrasting AVP effects are mediated by $\mathrm{V} 1 \mathrm{a}$ receptors, the $\mathrm{V} 1 \mathrm{a}$ receptor-selective agonist was tested on AMPA currents. In AVP neurons, F-180 reduced AMPA currents by $33.1 \pm 7.8 \%(p<0.05)$ in four of six cells, an effect similar to that of AVP (Fig. $4 B, C$ ). One cell showed no change, and the other increased the AMPA current by $53.8 \%$. In 
A1

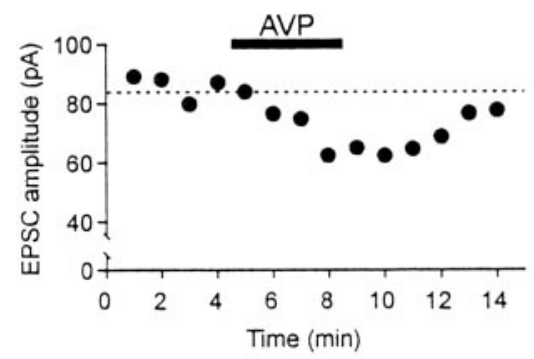

B1

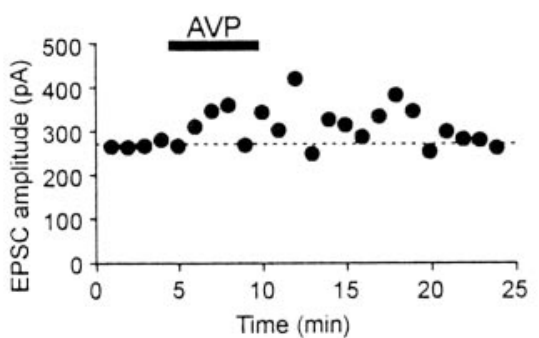

$\mathrm{A} 2$

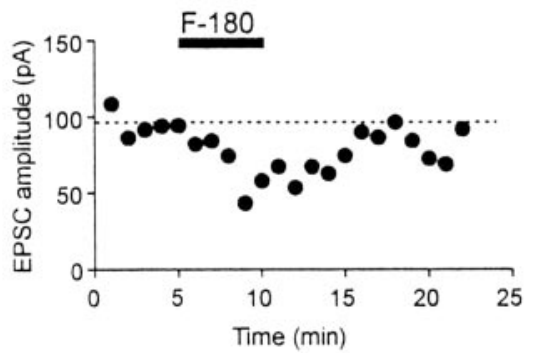

B2

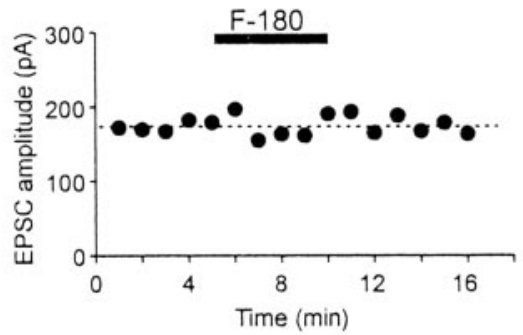

A3

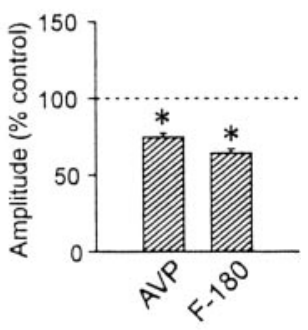

B3

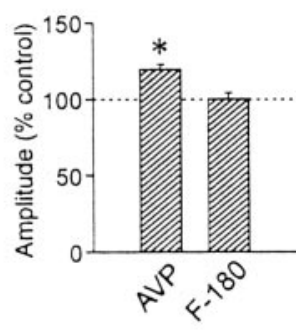

Figure 2. AVP differentially modulates the amplitude of the evoked EPSCs in the two types of magnocellular neurons. A1, Time-dependent plot of pharmacologically isolated EPSCs recorded from a typical AVP neuron. Each closed circle represents an average of 3- 6 consecutive EPSCs elicited per minute. Bath application of AVP ( $2 \mu \mathrm{m})$ reversibly reduces the amplitude of the EPSCs. A2, The inhibitory effect of AVP was mimicked by the V1a receptor agonist F-180. A3, Summary of the effect of AVP and F-180 on EPSCs recorded from AVP neurons. B1, Time-dependent plot of evoked EPSCs in a representative OXT neuron. Bath application of AVP $(2 \mu \mathrm{M})$ reversibly enhances the amplitude of the EPSCs. B2, In contrast to AVP neurons, F-180 has no effect on evoked EPSCs recorded in OXT neurons. B3, Summary of the effect of AVP and F-180 on EPSCs recorded from OXT neurons. ${ }^{*} p<0.05$.

\section{AVP neuron}
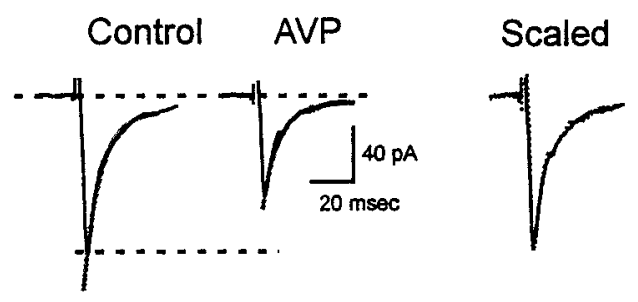

\section{OXT neuron}
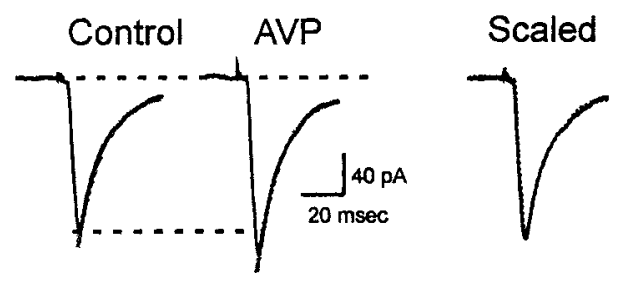

Figure 3. Decay time constant is not altered by AVP. The decay time constant of evoked EPSCs recorded in a representative AVP neuron (top traces) or an OXT neuron (bottom traces) does not change in the presence of AVP, as evident from scaled, superimposed traces at right.

contrast to the above AVP result, OXT neurons showed no response to F-180 in six of seven cells tested $(-3.5 \pm 4.8 \% ; p>$ $0.05)$, with one cell showing a $15.9 \%$ reduction. Similar to the EPSC result, there was no significant correlation between the control AMPA current and the direction of the response to AVP or F-180 $\left(R^{2}=0.11\right.$; slope deviation, $\left.p>0.05\right)$. To further confirm this observation, the V1a receptor-specific antagonist SR49059 $(10 \mu \mathrm{M})$ was applied for $5 \mathrm{~min}$ before AVP application, and it blocked the inhibition of AMPA current by AVP in AVP neurons (AVP alone, $-25.0 \pm 8.9 \%$ vs V1a antagonist plus AVP, $7.8 \pm 2.5 \% ; p<0.05 ; n=4$ ) (Fig. $4 A, C$ ). Thus, the effect of AVP on AMPA currents is mediated by V1a receptors in AVP neurons but not in OXT neurons.

Previous studies on neuropeptidergic receptors suggest that in MCNs, V1a receptors are located exclusively on AVP neurons and OXT receptors are expressed in OXT neurons (FreundMercier et al., 1994; Hurbin et al., 1998). Because AVP can bind to OXT receptors as well as to $\mathrm{V} 1$ a receptors (Muhlethaler and Dreifuss, 1983), a possibility exists that the facilitatory effect of AVP on AMPA current in OXT neurons may be mediated by OXT receptors. To test this idea, a selective OXT receptor antagonist, [des-glycinamide ${ }^{9}, d\left(\mathrm{CH}_{2}\right)_{5}{ }^{1}, \mathrm{O}-\mathrm{Me}-\mathrm{Tyr}^{2}, \mathrm{Try}^{4}, \mathrm{Orn}^{8}$ ]-vasotocin $(10 \mu \mathrm{M})$ was used. Pretreatment of the slices with this OXT antagonist for $5 \mathrm{~min}$ followed by AVP resulted in a blockade of the AVP-induced AMPA current facilitation in OXT neurons (AVP alone, $21.5 \pm 8.0 \%$ vs OXT antagonist plus AVP, $-2.8 \pm 9.4 \%$; $p<0.05 ; n=5)$ (Fig. 5A,C). In addition, OXT (500 nм to $1 \mu \mathrm{M}$ ) induced a similar increase in AMPA current in this type of cell $(14.5 \pm 3.9 \% ; p<0.05 ; n=5)$ (Fig. $5 B, C$ ). This result indicates that in OXT neurons, AVP exerts its effect through activation of OXT receptors. By contrast, in AVP neurons, pretreatment with OXT antagonist had no effect on AVP-induced inhibition of AMPA currents (AVP alone, $54.6 \pm 6.7 \%$ reduction vs OXT antagonist plus AVP, $52.7 \pm 6.1 \%$ reduction; $p>0.05 ; n=7$ ), further supporting the finding that AVP depresses excitatory transmission through V1a receptors in this type of neuron.

\section{Discussion}

The present study demonstrates that AVP modulates excitatory synaptic transmission differentially in different subpopulations 

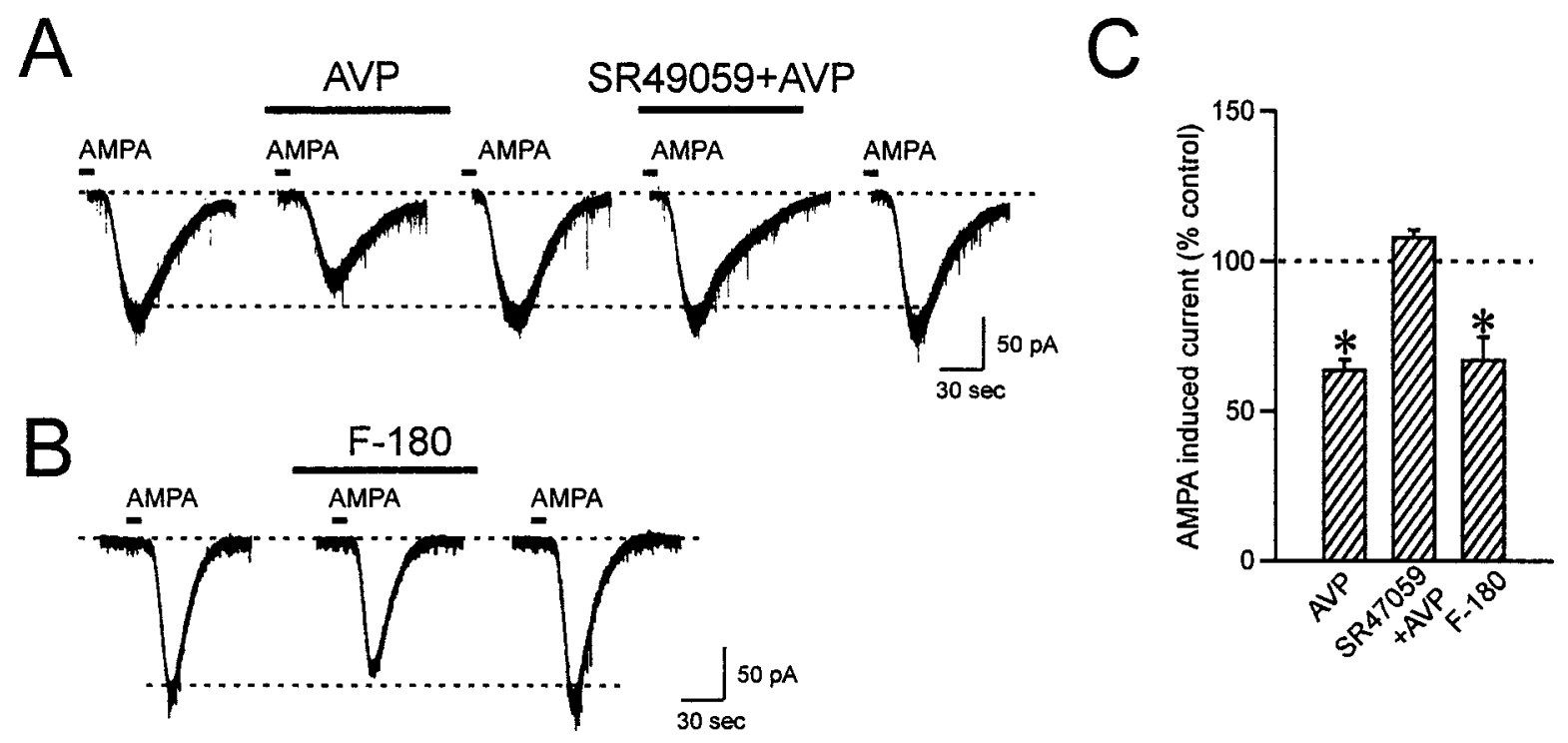

Figure 4. AVP inhibits AMPA-induced currents in AVP neurons. A, AVP reduces the inward currents induced by brief bath application of AMPA in AVP neurons. This inhibition is blocked by the V1a receptor specific antagonist SR49059. B, The inhibitory effect of AVP is mimicked by F-180. C, Summary bar graph showing the changes in AMPA-induced currents compared with control. ${ }^{*} p<0.05$.
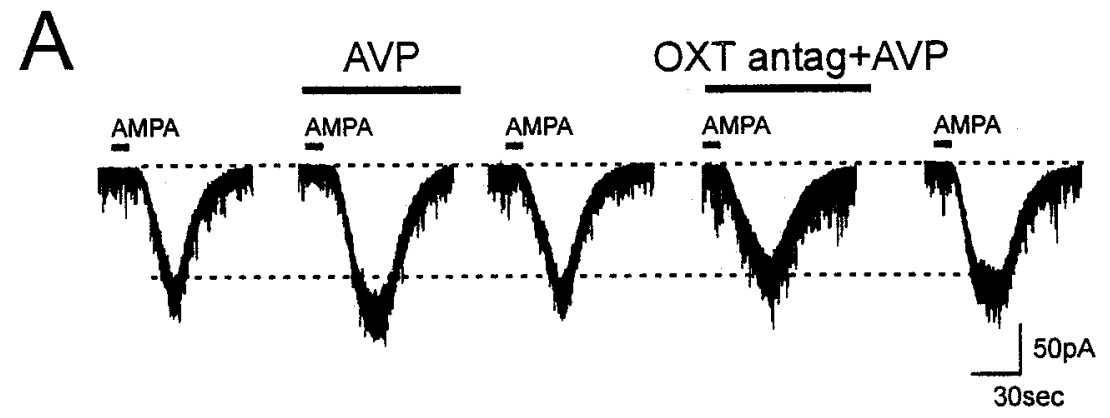

B

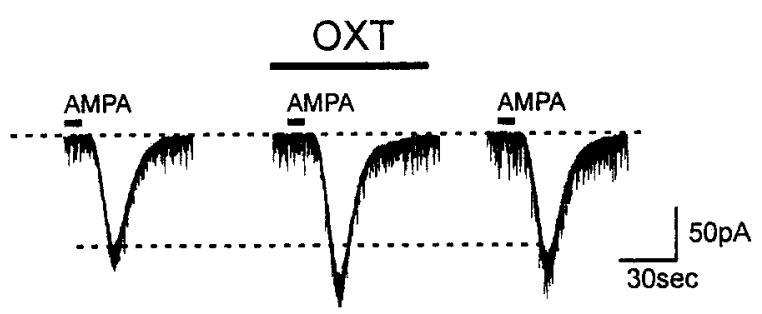

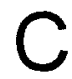

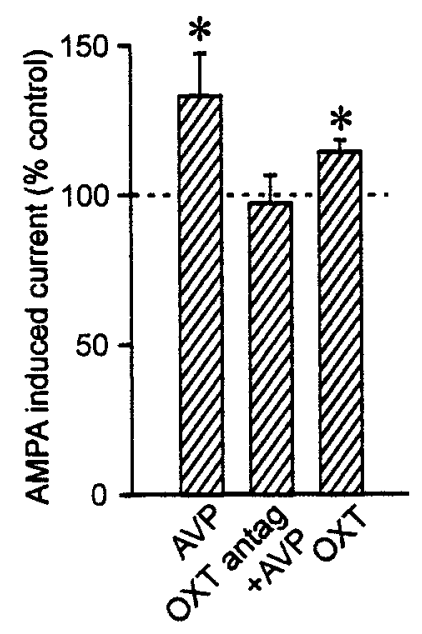

Figure 5. AVP facilitates AMPA-induced currents in OXT neurons. A, AVP increases the AMPA-induced inward currents in OXT neurons. This facilitation is blocked by the OXT receptor-specific antagonist [des-glycinamide ${ }^{9}, d\left(\mathrm{CH}_{2}\right)_{5}{ }^{1}, 0$-Me-Tyr ${ }^{2}, \mathrm{Tyr}^{4}, 0 \mathrm{rn}^{8}$ ]-vasotocin. $B$, OXT has a similar effect on AMPA-induced currents in OXT neurons. $C$, Summary bar graph showing the changes in AMPA-induced currents compared with control. * $p<0.05$.

of supraoptic MCNs. Two subpopulations of MCNs were characterized by the presence or absence of hyperpolarizationinduced IR and its coexistence with an SOR, a current found previously in most OXT-immunoreactive neurons (Stern and Armstrong, 1995). We showed that a combination of these currents was a highly reliable fingerprint for identifying MCN phenotype on the basis of correlative immunohistochemistry. Identification of the phenotype of recorded cells has been difficult with perforated patch-clamp recording because of lack of access of dyes into the cytosol. We resolved this problem by modifying the single-cell labeling method used for dye-filling a neuron simultaneously with juxtacellular recording. Briefly, by modulating the firing of a neuron with low-intensity positive-current injection through a microelectrode, biocytin or neurobiotin can be filled into the cell, presumably by electroporation (Pinault,
1996; Kirouac and Pittman, 1999). In our preparation, perforated-patch recordings were performed, after which lowintensity positive-current was injected to cause the cells to fire at $6-10 \mathrm{~Hz}$. Biocytin dissolved in the recording solution in the patch pipette was introduced successfully into the recorded neurons by this method. Thus, there is now the potential for phenotypic identification of neurons on which electrophysiological studies have been performed by perforated-patch-clamp recording. This is especially relevant for studying phenomena that involve postsynaptic cytosolic component(s), which by nature require the use of perforated-patch over conventional whole-cell recording. Using this technique, we revealed that MCNs that show SOR/IR are OXT neurons, and those that do not show SOR/IR are AVP neurons. By these criteria, we showed that AVP enhanced the EPSCs in most OXT neurons, whereas it depressed 
EPSCs in AVP neurons. These contrasting results seem to result from AVP acting on different receptors.

Our previous report suggests that endogenous neuropeptides were released from MCNs, which induced a 30-40\% reduction in evoked EPSC amplitude (Kombian et al., 1997). The magnitude of this inhibition was equivalent to that obtained by $1-10 \mu \mathrm{M}$ OXT (Kombian et al., 1997) and by 2-5 $\mu$ M AVP (Kombian et al., 2000). Thus, it is likely that during intense MCN activities, the concentration of dendritically released neuropeptide in the extracellular space may reach the micromolar range. In addition, another group has reported that OXT shows its effect on IPSCs at 1-10 $\mu \mathrm{M}$ ranges in slice preparation (Brussaard et al., 1996). However, endogenous peptidase activity significantly reduces the concentration of neuropeptides by up to 100-fold (Saleh et al., 1996). Therefore, the concentration that effectively reaches the receptors is probably in the submicromolar range, close to the $\mathrm{ED}_{50}$ concentration that activates isolated cells (Lambert et al., 1994; Dayanithi et al., 1996).

\section{Voltage-dependent currents characterize two distinct populations of magnocellular neurons}

MCNs can be distinguished from other neurons by their characteristic delayed onset of action potential firing to depolarization from hyperpolarized potentials (Tasker et al., 1991; Armstrong, 1995). In addition, two subtypes of MCNs, namely AVP and OXT neurons, have been characterized by a number of electrophysiological features, including SOR (Armstrong et al., 1994; Stern and Armstrong, 1995). We identified another characteristic that may be used for identification of the two subpopulations: one population had a linear current-voltage relationship in response to hyperpolarizing voltage steps from $-40 \mathrm{mV}$, and the other showed an IR at hyperpolarized potential, a response often associated with an SOR. The present study has demonstrated that MCNs that showed both IR and SOR were immunohistochemically identified as OXT neurons, whereas other MCNs that did not display either IR or SOR were identified as AVP neurons. Thus, we suggest that the occurrence of both of these currents can be used as a reliable criterion to characterize the phenotype of MCNs in the SON.

The IR recorded in this study is unlikely to correspond to $I_{\mathrm{H}}$, which is not activated at the early time points seen in the present experiments (Erickson et al., 1993; Ghamari-Langroudi and Bourque, 2000). Although it could correspond to the cesiumsensitive current revealed after blockade of $I_{\mathrm{H}}$ (GhamariLangroudi and Bourque, 2001), the latter current was seen in all cells tested, whereas ours was seen in only half of the cells. Thus, we remain open to the possibility that this rectification actually represents the deactivation of steady-state potassium current, whatever its underlying mechanism. Because the object of the present study is to investigate the synaptic action of AVP, we have not characterized this current further.

\section{AVP differentially modulates EPSCs in different populations of magnocellular neurons}

In the present study, we have identified two distinct actions of AVP, i.e., AVP decreases evoked EPSC amplitudes in AVP neurons, whereas it increases EPSC amplitudes in OXT neurons. AVP induces these differential effects via different postsynaptic receptors.

A postsynaptic action of AVP was clearly demonstrated in both cell populations, because AVP changed the amplitude of AMPA-induced inward currents, which is a measure of postsynaptic non-NMDA receptor sensitivity. AVP seemed to modulate
non-NMDA receptors to increase or decrease their sensitivity in OXT or AVP neurons, respectively. These opposing effects were not accompanied by alteration in EPSC decay constant, indicating that a change in desensitization of receptors did not underlie the effects. Rather, it was a result of alteration of steady-state currents, as shown by changes in the amplitudes of AMPAinduced steady-state currents. Different postsynaptic neuropeptide receptors seem to be responsible for the differential effects of AVP. In AVP neurons, V1a receptors mediate the decrease in AMPA current, because the AVP effect was blocked by V1a receptor antagonist and mimicked by V1a agonist. In OXT neurons, OXT receptors play this role, as evidenced by blockade of AMPA current facilitation by OXT receptor antagonist, whereas a V1a receptor agonist did not mimic AVP effect. This is in agreement with a previous report that showed clearly that AVP neurons express V1a receptors and OXT neurons express OXT receptors (Freund-Mercier et al., 1994; Hurbin et al., 1998). Although the exclusive expression of OXT receptors by OXT neurons has been found in female pregnant animals (FreundMercier et al., 1994), our study gives functional evidence that similar expression specification exists in male animals also. The mechanism(s) by which activation of OXT and V1a receptors modulates the sensitivity of non-NMDA receptors is not yet known. V1a and OXT receptors are closely related members of the G-protein receptor superfamily (Morel et al., 1992; Rozen et al., 1995); however, it is possible that the signal transduction pathways coupled to each of these receptor types are different in SON neurons. This is envisioned from the differential calcium dynamics resulting from activation of these two types of receptors: V1a receptor activation results in calcium entry via voltagegated calcium channels (Dayanithi et al., 1996; Sabatier et al., 1997), whereas OXT receptors induce calcium release from internal stores independently of calcium influx (Lambert et al., 1994). Also, differences in calcium buffering capacity between OXT and AVP neurons have been observed (Miyata et al., 1998). Such differences in calcium source and dynamics may result in differential calcium-dependent modulation of postsynaptic nonNMDA receptors. Alternatively, it has been shown that AVP and OXT neurons possess functionally different non-NMDA receptors, because of a differential combination of receptor subunits and/or their modulation (Stern et al., 1999). Different glutamate receptor subunits could be modulated differentially and contribute differently to the function of the receptors. The modulation of non-NMDA receptors seems to involve cytosolic constituents, because use of conventional whole-cell patch configuration, instead of perforated patch, abolished the AVP effect on AMPAinduced currents (our unpublished observations).

We have reported previously that OXT reduced EPSCs through a presynaptic action (Kombian et al., 1997). In that study, there was no evidence for a postsynaptic OXT receptor being responsible for OXT action on excitatory transmission. We believe that in our previous study, we sampled predominantly putative AVP neurons, because at that time we found little evidence for either SOR or IR reported here. As a result of technical modification in our slice preparation (change in $\mathrm{Na}^{+}$and $\mathrm{Ca}^{2+}$ concentration in the dissection solution), we now obtain substantially more cells with characteristics of OXT neurons. This change may be an indication that OXT neurons are more susceptible to excitotoxic insults, such as dissection. Our result supports such a possibility: AVP released within the nucleus would favor an excitation of OXT neurons while causing inhibition of AVP neurons. The activity of AVP neurons is tuned by AVP, so that the neurons would be quieted during high level of activity (Ludwig et 
al., 1997). In contrast, OXT that may also be released during high activity of the nucleus will further increase the activity of OXT neurons (Yamashita et al., 1987; Moos and Richard, 1989). Such a reaction discrepancy between OXT and AVP neurons during high levels of activity may underlie the differential survival in different media conditions.

\section{Physiological implications}

Our finding provides an underlying cellular mechanism for an inhibitory action of AVP on AVP neurons that would effectively limit the excitation of these neurons (Ludwig and Leng, 1997). Given that the plasma level of AVP has to change very little to cause maximal changes in urine osmolarity (Robertson, 1985), very high levels of activity of AVP neurons may not be necessary for normal physiological function. It is interesting that the MCNs are known to be relatively resistant to glutamate receptormediated excitotoxicity (Hu et al., 1992; Bains et al., 2001). EPSC inhibition caused by dendritic release of AVP could certainly contribute to this resistance.

MCNs in the SON are coupled through gap junctions (Andrew et al., 1981). Such characteristics may suggest that dendrites and/or somata of neighboring neurons may be in close apposition, so that neuropeptides released from one neuron may act on another neuron in a paracrine manner. Thus, the results of the present study that AVP has actions on both OXT and AVP neurons may be physiologically relevant. This phenomenon may become particularly evident during times of high activity when glial cells retract from interneuronal spaces, allowing easier diffusion of neuropeptides between neurons (Tweedle and Hatton, 1977; Chapman et al., 1986; Theodosis et al., 1986).

\section{References}

Andrew RD, MacVicar BA, Dudek FE, Hatton GI (1981) Dye transfer through gap junctions between neuroendocrine cells of rat hypothalamus. Science 211:1187-1189.

Armstrong WE (1995) Morphological and electrophysiological classification of hypothalamic supraoptic neurons. Prog Neurobiol 47:291-339.

Armstrong WE, Smith BN, Tian M (1994) Electrophysiological characteristics of immunochemically identified rat oxytocin and vasopressin neurones in vitro. J Physiol (Lond) 475:115-128.

Bains JS, Follwell MJ, Latchford KJ, Anderson JW, Ferguson AV (2001) Slowly inactivating potassium conductance $\left(I_{\mathrm{D}}\right)$ : a potential target for stroke therapy. Stroke 32:2624-2634.

Ben Barak Y, Russell JT, Whitnall MH, Ozato K, Gainer H (1985) Neurophysin in the hypothalamo-neurohypophysial system. I. Production and characterization of monoclonal antibodies. J Neurosci 5:81-97.

Brussaard AB, Kits KS, de Vlieger TA (1996) Postsynaptic mechanism of depression of GABAergic synapses by oxytocin in the supraoptic nucleus of immature rat. J Physiol (Lond) 497:495-507.

Chapman DB, Theodosis DT, Montagnese C, Poulain DA, Morris JF (1986) Osmotic stimulation causes structural plasticity of neurone-glia relationships of the oxytocin but not vasopressin secreting neurones in the hypothalamic supraoptic nucleus. Neuroscience 17:679-686.

Dayanithi G, Widmer H, Richard P (1996) Vasopressin-induced intracellular $\mathrm{Ca}^{2+}$ increase in isolated rat supraoptic cells. J Physiol (Lond) 490:713-727.

Erickson KR, Ronnekleiv OK, Kelly MJ (1993) Role of a T-type calcium current in supporting a depolarizing potential, damped oscillations, and phasic firing in vasopressinergic guinea pig supraoptic neurons. Neuroendocrinology 57:789-800.

Freund-Mercier MJ, Stoeckel ME, Klein MJ (1994) Oxytocin receptors on oxytocin neurones: histoautoradiographic detection in the lactating rat. J Physiol (Lond) 480:155-161.

Ghamari-Langroudi M, Bourque CW (2000) Excitatory role of the hyperpolarization-activated inward current in phasic and tonic firing of rat supraoptic neurons. J Neurosci 20:4855-4863.

Ghamari-Langroudi M, Bourque CW (2001) Ionic basis of the caesium- induced depolarisation in rat supraoptic nucleus neurones. J Physiol (Lond) 536:797-808.

Gouzenes L, Desarmenien MG, Hussy N, Richard P, Moos FC (1998) Vasopressin regularizes the phasic firing pattern of rat hypothalamic magnocellular vasopressin neurons. J Neurosci 18:1879-1885.

Hirasawa M, Kombian SB, Pittman QJ (2001) Oxytocin retrogradely inhibits evoked, but not miniature, EPSCs in the rat supraoptic nucleus: role of N- and P/Q-type calcium channels. J Physiol (Lond) 532:595-607.

Hu B, Cunningham JT, Nissen R, Renaud LP, Bourque CW (1992) Rat supraoptic neurons are resistant to glutamate neurotoxicity. NeuroReport 3:87-90.

Hurbin A, Boissin-Agasse L, Orcel H, Rabie A, Joux N, Desarmenien MG, Richard P, Moos FC (1998) The V1a and V1b, but not V2, vasopressin receptor genes are expressed in the supraoptic nucleus of the rat hypothalamus, and the transcripts are essentially colocalized in the vasopressinergic magnocellular neurons. Endocrinology 139:4701-4707.

Kirouac GJ, Pittman QJ (1999) Identification of barosensitive neurons in the mediobasal forebrain using juxtacellular labeling. Am J Physiol 276:R1766-R1771.

Kombian SB, Zidichouski JA, Pittman QJ (1996) GABA $_{B}$ receptors presynaptically modulate excitatory synaptic transmission in the rat supraoptic nucleus in vitro. J Neurophysiol 76:1166-1179.

Kombian SB, Mouginot D, Pittman QJ (1997) Dendritically released peptides act as retrograde modulators of afferent excitation in the supraoptic nucleus in vitro. Neuron 19:903-912.

Kombian SB, Mouginot D, Hirasawa M, Pittman QJ (2000) Vasopressin preferentially depresses excitatory over inhibitory synaptic transmission in the rat supraoptic nucleus in vitro. J Neuroendocrinol 12:361-367.

Lambert RC, Dayanithi G, Moos FC, Richard P (1994) A rise in the intracellular $\mathrm{Ca}^{2+}$ concentration of isolated rat supraoptic cells in response to oxytocin. J Physiol (Lond) 478:275-287.

Leng G, Brown CH, Russell JA (1999) Physiological pathways regulating the activity of magnocellular neurosecretory cells. Prog Neurobiol 57:625-655.

Li Z, Hatton GI (2000) Histamine suppresses non-NMDA excitatory synaptic currents in rat supraoptic nucleus neurons. J Neurophysiol 83:2616-2625.

Ludwig M, Leng G (1998) Intrahypothalamic vasopressin release: an inhibitor of systemic vasopressin secretion? Adv Exp Med Biol 449:163-173.

Ludwig M, Brown CH, Russell JA, Leng G (1997) Local opioid inhibition and morphine dependence of supraoptic nucleus oxytocin neurones in the rat in vivo. J Physiol (Lond) 505:145-152.

Mason WT, Hatton GI, Ho YW, Chapman C, Robinson IC (1986) Central release of oxytocin, vasopressin and neurophysin by magnocellular neurone depolarization: evidence in slices of guinea pig and rat hypothalamus. Neuroendocrinology 42:311-322.

Miyata S, Khan AM, Hatton GI (1998) Colocalization of calretinin and calbindin-D28k with oxytocin and vasopressin in rat supraoptic nucleus neurons: a quantitative study. Brain Res 785:178-182.

Moos F, Richard P (1989) Paraventricular and supraoptic bursting oxytocin cells in rat are locally regulated by oxytocin and functionally related. J Physiol (Lond) 408:1-18.

Moos F, Freund-Mercier MJ, Guerne Y, Guerne JM, Stoeckel ME, Richard P (1984) Release of oxytocin and vasopressin by magnocellular nuclei in vitro: specific facilitatory effect of oxytocin on its own release. J Endocrinol 102:63-72.

Morel A, O'Carroll AM, Brownstein MJ, Lolait SJ (1992) Molecular cloning and expression of a rat V1a arginine vasopressin receptor. Nature 356:523-526.

Muhlethaler M, Dreifuss JJ (1983) Excitation of hippocampal neurones by posterior pituitary peptides: vasopressin and oxytocin compared. Prog Brain Res 60:147-151.

Oliet SH, Poulain DA (1999) Adenosine-induced presynaptic inhibition of IPSCs and EPSCs in rat hypothalamic supraoptic nucleus neurones. J Physiol (Lond) 520:815-825.

Pinault D (1996) A novel single-cell staining procedure performed in vivo under electrophysiological control: morpho-functional features of juxtacellularly labeled thalamic cells and other central neurons with biocytin or neurobiotin. J Neurosci Methods 65:113-136.

Pow DV, Morris JF (1989) Dendrites of hypothalamic magnocellular neurons release neurohypophysial peptides by exocytosis. Neuroscience $32: 435-439$. 
Robertson GL (1985) Osmoregulation of thirst and vasopressin secretion: functional properties and their relationship to water balance. In: Vasopressin (Schrier RW, ed), pp 203-212. New York: Raven.

Rozen F, Russo C, Banville D, Zingg HH (1995) Structure, characterization, and expression of the rat oxytocin receptor gene. Proc Natl Acad Sci USA 92:200-204.

Sabatier N, Richard P, Dayanithi G (1997) L-, N- and T- but neither P- nor Q-type $\mathrm{Ca}^{2+}$ channels control vasopressin-induced $\mathrm{Ca}^{2+}$ influx in magnocellular vasopressin neurones isolated from the rat supraoptic nucleus. J Physiol (Lond) 503:253-268.

Saleh TM, Kombian SB, Zidichouski JA, Pittman QJ (1996) Peptidergic modulation of synaptic transmission in the parabrachial nucleus in vitro: importance of degradative enzymes in regulating synaptic efficacy. J Neurosci 16:6046-6055.

Schrader LA, Tasker JG (1997) Presynaptic modulation by metabotropic glutamate receptors of excitatory and inhibitory synaptic inputs to hypothalamic magnocellular neurons. J Neurophysiol 77:527-536.

Stern JE, Armstrong WE (1995) Electrophysiological differences between oxytocin and vasopressin neurones recorded from female rats in vitro. J Physiol 488:701-708.
Stern JE, Galarreta M, Foehring RC, Hestrin S, Armstrong WE (1999) Differences in the properties of ionotropic glutamate synaptic currents in oxytocin and vasopressin neuroendocrine neurons. J Neurosci 19:3367-3375.

Tasker JG, Hoffman NW, Dudek FE (1991) Comparison of three intracellular markers for combined electrophysiological, morphological and immunohistochemical analyses. J Neurosci Methods 38:129-143.

Theodosis DT, Chapman DB, Montagnese C, Poulain DA, Morris JF (1986) Structural plasticity in the hypothalamic supraoptic nucleus at lactation affects oxytocin-, but not vasopressin-secreting neurones. Neuroscience 17:661-678.

Tweedle CD, Hatton GI (1977) Ultrastructural changes in rat hypothalamic neurosecretory cells and their associated glia during minimal dehydration and rehydration. Cell Tissue Res 181:59-72.

Vyklicky Jr L, Patneau DK, Mayer ML (1991) Modulation of excitatory synaptic transmission by drugs that reduce desensitization at AMPA/kainate receptors. Neuron 7:971-984.

Yamashita H, Okuya S, Inenaga K, Kasai M, Uesugi S, Kannan H, Kaneko T (1987) Oxytocin predominantly excites putative oxytocin neurons in the rat supraoptic nucleus in vitro. Brain Res 416:364-368. 